\title{
Johor Strait as a hotspot for trace elements contamination in Peninsular Malaysia.
}

\begin{abstract}
Present study was conducted to evaluate current status of trace elements contamination in the surface sediments of the Johor Strait. Iron $(2.54 \pm 1.24 \%)$ was found as the highest occurring element, followed by those of zinc $(210.45 \pm 115.4 \mu \mathrm{g} / \mathrm{g})$, copper $(57.84 \pm 45.54 \mu \mathrm{g} / \mathrm{g})$, chromium $(55.50 \pm 31.24 \mu \mathrm{g} / \mathrm{g})$, lead $(52.52 \pm 28.41 \mu \mathrm{g} / \mathrm{g})$, vanadium $(47.76 \pm 25.76 \mu \mathrm{g} / \mathrm{g})$, arsenic $(27.30 \pm 17.11 \mu \mathrm{g} / \mathrm{g})$, nickel $(18.31 \pm 11.77 \mu \mathrm{g} / \mathrm{g})$, cobalt $(5.13 \pm 3.12 \mu \mathrm{g} / \mathrm{g})$, uranium $(4.72 \pm 2.52 \mu \mathrm{g} / \mathrm{g})$, and cadmium $(0.30 \pm 0.30 \mu \mathrm{g} / \mathrm{g})$, respectively. Bioavailability of cobalt, nickel, copper, zinc, arsenic and cadmium were higher than $50 \%$ of total concentration. Vanadium, copper, zinc, arsenic and cadmium were found significantly different between the eastern and western part of the strait $(\mathrm{p}<0.05)$. Combining with other factors, Johor Strait is suitable as a hotspot for trace elements contamination related studies.
\end{abstract}

Keyword: Johor Strait; Sediments; Trace elements; Bioavailability; Hotspot. 